\title{
Geodesic motion in the Kundt spacetimes and the character of envelope singularity
}

\author{
J. Podolský* and M. Beláñ ${ }^{\dagger}$ \\ Institute of Theoretical Physics, Charles University in Prague, \\ V Holešovičkách 2, 18000 Prague 8, Czech Republic.
}

November 16, 2018

\begin{abstract}
We investigate geodesics in specific Kundt type $N$ (or conformally flat) solutions to Einstein's equations. Components of the curvature tensor in parallelly transported tetrads are then explicitly evaluated and analyzed. This elucidates some interesting global properties of the spacetimes, such as an inherent rotation of the wave-propagation direction, or the character of singularities. In particular, we demonstrate that the characteristic envelope singularity of the rotated wave-fronts is a (non-scalar) curvature singularity, although all scalar invariants of the Riemann tensor vanish there.
\end{abstract}

PACS: 04.20.Jb; 04.30.Nk

\section{Introduction}

In 1961 a general class of solutions to Einstein's equations which admit a non-expanding, shearfree, and twist-free null congruence was introduced and described by Wolfgang Kundt [1-3] (for recent reviews see, e.g., [4], [5]). This contained some previously known families, in particular the $p p$-waves [6-9] or the Nariai [10] and Bertotti-Robinson [11-13] universes. Within his large class, Kundt also identified new solutions of various types, among them specific type $N$ spacetimes representing plane-fronted gravitational waves which were geometrically different from famous $p p$-waves. These so-called Kundt waves are either vacuum solutions or contain pure radiation. Their generalization to a non-vanishing value of the cosmological constant $\Lambda$ was later found by Ozsváth et al [14] (see also [15-18]). Very recently, these solutions have been further generalized in [19] where a complete family of Kundt waves of type III (which admit $\Lambda$ and/or pure radiation) has been derived and classified. Geometry of the wave surfaces and their envelope singularity has also been analyzed. For particular choices of the metric functions one recovers more special type $N$ Kundt waves and conformally flat non-expanding spacetimes.

In fact, the family of conformally flat pure radiation Kundt metrics attracted attention few years ago [20-27]. In general, they contain no invariants or Killing or homothetic vectors, and thus provide an interesting exceptional case for invariant classification of exact solutions. It was demonstrated by Skea [25] that, to distinguish the Wils [20] metric within the more general Edgar-Ludwig solution [22], it is necessary to go as far as but no further than the fourth

\footnotetext{
${ }^{*}$ E-mail: podolsky@mbox.troja.mff.cuni.cz

${ }^{\dagger} \mathrm{E}$-mail: halef@atrey.karlin.mff.cuni.cz
} 
derivative of the curvature tensor. This result supports the conjecture that any space-time can be uniquely characterized using the derivatives of the curvature tensor in which no higher than the fourth derivative is ever required.

Even more interestingly, it has been recently demonstrated $[28,29]$ that for the above Kundt spacetimes all curvature invariants of all orders identically vanish. They may thus play an important role in string theory and quantum gravity since there are no quantum corrections to all perturbative orders [30].

Despite the fact that particular aspects of these spacetimes have been studied for decades, many questions concerning their geometrical and physical properties still remain open. For example, analysis of the geodesic deviation [31] revealed the structure of relative motions of test particles in the Kundt gravitational waves but it is not yet clear whether the geodesic motion itself does exhibit a chaotic behaviour, analogous to the chaos in $p p$-waves [32].

We consider here vacuum Kundt type $N$ waves but some results also apply to conformally flat pure radiation solutions of this non-expanding class. The purpose of our paper is to contribute to understanding of their global structure, namely the character of singularities. For principal reasons this is not an easy task since all curvature invariants - which would unambiguously identify physical singularities - vanish. Thus, to analyse singularities in the Kundt spacetimes (summarized here in section 2) it is necessary to study the behaviour of free test particles, which is done in section 3 . In the subsequent section 4 we find frames parallelly transported along timelike and null geodesics, and in section 5 we project the curvature tensor onto these tetrads. We employ these results for discussion of the singularities in the final section 6 .

\section{The Kundt spacetimes}

The metric of type $N$ or conformally flat Kundt spacetimes, which are either vacuum or contain pure radiation, can be written in the form (see, e.g., [5])

$$
\begin{gathered}
\mathrm{d} s^{2}=2 \mathrm{~d} \zeta \mathrm{d} \bar{\zeta}-2 Q^{2} \mathrm{~d} u \mathrm{~d} v+F \mathrm{~d} u^{2}, \\
Q=\zeta+\bar{\zeta}, \quad F=2 Q^{2} v^{2}-Q H,
\end{gathered}
$$

where $H(\zeta, \bar{\zeta}, u)$ is a function of the spatial coordinates $\zeta, \bar{\zeta}$, and of the retarded time $u$. A simple transformation $v=w Q^{-2}$ puts the metric into the Kundt canonical form $[1,4]$

$$
\mathrm{d} s^{2}=2 \mathrm{~d} \zeta \mathrm{d} \bar{\zeta}-2 \mathrm{~d} u(\mathrm{~d} w+W \mathrm{~d} \zeta+\bar{W} \mathrm{~d} \bar{\zeta}+\mathcal{H} \mathrm{d} u)
$$

with $W=-2 w Q^{-1}, \mathcal{H}=-w^{2} Q^{-2}+\frac{1}{2} Q H$ (the $p p$-waves corresponding to $W=0$ and $\mathcal{H}=H$ in (2) will not be investigated here). In the natural null tetrad

$$
\mathbf{k}=\partial_{v}, \quad \mathbf{l}=(F / 2) Q^{-4} \partial_{v}+Q^{-2} \partial_{u}, \quad \mathbf{m}=\partial_{\bar{\zeta}}, \quad \overline{\mathbf{m}}=\partial_{\zeta},
$$

( $\mathbf{k}$ is the quadruple principal null direction) the only non-vanishing Weyl and Ricci scalars are

$$
\Psi_{4}=\frac{1}{2} Q^{-3} H_{, \zeta \zeta}, \quad \Phi_{22}=\frac{1}{2} Q^{-3} H_{, \zeta \bar{\zeta}} .
$$

The spacetimes are thus conformally flat when $H_{, \zeta \zeta}=0$, i.e. if $H$ has a special form

$$
H_{0}=A_{0}+A_{1} \zeta+\bar{A}_{1} \bar{\zeta}+A_{2} \zeta \bar{\zeta}
$$

where $A_{i}$ are arbitrary complex functions of $u$. These spacetimes containing pure radiation characterized by $\Phi_{22}=A_{2} /\left(2 Q^{3}\right)$ were considered by Kundt [1-3] and others [20-27]. For $A_{2}=0$ 

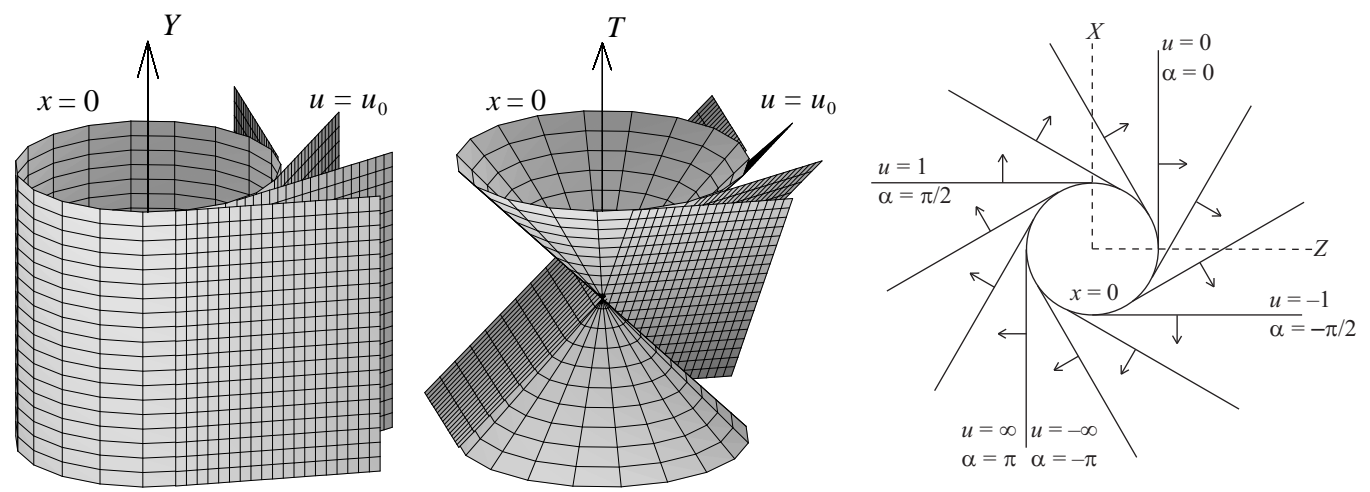

Figure 1: The wave surfaces $u=u_{0}=\tan (\alpha / 2)$ with $x \geq 0$ are tangent half-planes to expanding cylinder (for $T=$ const.; Left), cone (for $Y=$ const.; Middle), or circle (for $T, Y=$ const.; Right) in Minkowski background coordinates. At any time $T$ each wave surface is bounded by the line where it touches the cylinder $x=0$. For different values of $\alpha$, the successive surfaces are rotated around the cylinder centered on the $Y$-axis which expands with the speed of light (four surfaces $u=u_{0}$ are indicated; Left and Middle). As the circular caustic envelope $x=0$ expands (Right), the tangent half-planes propagate in the spatial direction perpendicular to each wave surface.

the matter is absent, and $H_{0}$ can be set zero by a suitable coordinate transformation [18]. Such solution with $H=0$ corresponds to flat Minkowski space, see transformation (11) below.

Vacuum type $N$ Kundt waves arise when $\Phi_{22}=0, \Psi_{4} \neq 0$, i.e. for

$$
H=f(\zeta, u)+\bar{f}(\bar{\zeta}, u),
$$

where $f$ is an arbitrary function of $\zeta$ and $u$, holomorphic in $\zeta$, such that $f_{, \zeta \zeta} \neq 0$. These solutions (generalized also to admit a cosmological constant $\Lambda$ ) were presented in $[14,15,18,19]$.

For our analysis of geodesics and singularities it is convenient to rewrite the Kundt solutions in real spatial coordinates $x$ and $y$, related to the complex coordinate $\zeta$ by

$$
\zeta=(x+\mathrm{i} y) / \sqrt{2} .
$$

The metric (11) obviously takes the form

$$
\mathrm{d} s^{2}=\mathrm{d} x^{2}+\mathrm{d} y^{2}-4 x^{2} \mathrm{~d} u \mathrm{~d} v+4\left(x^{2} v^{2}+x G\right) \mathrm{d} u^{2},
$$

where $G=-H /(2 \sqrt{2})$. For vacuum spacetimes the function $G(x, y, u)$ satisfies the Laplace equation, $G_{, x x}+G_{, y y}=0$. The Christoffel symbols in coordinates $(v, x, y, u) \equiv(0,1,2,3)$ are

$$
\begin{aligned}
& \Gamma_{01}^{0}=1 / x, \quad \Gamma_{03}^{0}=-2 v, \quad \Gamma_{13}^{0}=-(G / x)_{, x}, \quad \Gamma_{23}^{0}=-(G / x)_{, y}, \\
& \Gamma_{33}^{0}=4 v^{3}+4 v G / x-(G / x)_{, u}, \quad \Gamma_{33}^{1}=-4 x v^{2}-2 G-2 x G_{, x}, \\
& \Gamma_{03}^{1}=2 x, \quad \Gamma_{33}^{2}=-2 x G_{, y}, \quad \Gamma_{13}^{3}=1 / x, \quad \Gamma_{33}^{3}=2 v,
\end{aligned}
$$

and all independent non-vanishing components of the curvature tensor read

$$
R_{1313}=-2 x G_{, x x}, \quad R_{1323}=-2 x G_{, x y}, \quad R_{2323}=-2 x G_{, y y} .
$$

Let us also recall the geometry of the Kundt spacetimes in the weak field limit. It has been shown previously [26] that the transformation

$$
\begin{aligned}
X & =x(1+2 u v), & Y & =y, \\
Z & =x[v-u(1+u v)], & & T=x[v+u(1+u v)],
\end{aligned}
$$


puts the Kundt metric (8) to

$$
\mathrm{d} s^{2}=\mathrm{d} s_{0}^{2}+\frac{x G(x, y, u)}{X^{2}+Z^{2}-T^{2}}\left[\left(1+u^{2}\right) \mathrm{d} T-2 u \mathrm{~d} X-\left(1-u^{2}\right) \mathrm{d} Z\right]^{2}
$$

where $\mathrm{d} s_{0}^{2}=-\mathrm{d} T^{2}+\mathrm{d} X^{2}+\mathrm{d} Y^{2}+\mathrm{d} Z^{2}$ is the standard form of flat space corresponding to $G=0$. In (12), $x, y$ and $u$ should be expressed using the inverse of (11), namely

$$
\begin{array}{ll}
x= \pm \sqrt{X^{2}+Z^{2}-T^{2}}, & y=Y, \\
u=\frac{X \mp \sqrt{X^{2}+Z^{2}-T^{2}}}{T+Z}, & v= \pm \frac{T+Z}{2 \sqrt{X^{2}+Z^{2}-T^{2}}} .
\end{array}
$$

In the weak field limit (for small $G$ ) when the metric (12) is a perturbation of Minkowski background [26], each wave surface $u=u_{0}=$ const. corresponds to a hyperplane

$$
-T+\sin \alpha X+\cos \alpha Z=0, \quad Y \text { arbitrary },
$$

where $\alpha=2 \arctan u_{0}$. As $u_{0}$ increases from $-\infty$ to $+\infty$ then $\alpha$ goes from $-\pi$ to $+\pi$, and these wave surfaces form a family of null hyperplanes which rolls all around the cylinder

$$
X^{2}+Z^{2}=T^{2}, \quad Y \text { arbitrary }
$$

For $T>0$ its radius is expanding at the speed of light. In fact, it follows from (13) that this caustic surface, formed as the envelope of all rotated wave surfaces, corresponds to a singularity $x=0$ in the metric (8), see also [33].

To obtain a unique foliation (14) of the region outside the expanding cylinder (15) by the wave surfaces $u=u_{0} \in(-\infty,+\infty)$, it is necessary to restrict these to half-hyperplanes. This is achieved by considering only the range $x \geq 0$ (or $x \leq 0$ ). In both cases the wave surfaces are localized outside the cylinder (15) given by $x=0$ which is formed as their envelope as indicated in figure 1. The restriction $x \geq 0$ also resolves the ambiguity in the signs of (13) which corresponds to the invariance of the metric (8) under the reflections $x \rightarrow-x, G \rightarrow-G$.

Natural questions arise concerning the character of the envelope $x=0$ of the rotating family of wave fronts, and the region inside it which is not covered by the Kundt coordinates. Is this envelope singularity only a coordinate one or is it a "physical" singularity? Is it possible to extend geodesics from the outside region across $x=0$ ? And what tidal forces would the corresponding geodesic observers feel? We concentrate on these open problems in our paper.

\section{Geodesics in the Kundt spacetimes}

Considering the Christoffel symbols (9) the geodesic equations read

$$
\begin{aligned}
& \ddot{x}=-4 x \dot{v} \dot{u}+\left(4 x v^{2}+2 G+2 x G_{, x}\right) \dot{u}^{2}, \\
& \ddot{y}=2 x G_{, y} \dot{u}^{2}, \\
& \ddot{u}=-2(\dot{x} / x) \dot{u}-2 v \dot{u}^{2},
\end{aligned}
$$

(where the dot denotes differentiation with respect to an affine parameter $\tau$ ) plus a complicated equation for $\ddot{v}$. Instead of it, however, we take the normalisation condition of four-velocity:

$$
\dot{x}^{2}+\dot{y}^{2}-4 x^{2} \dot{u} \dot{v}+4 x^{2}\left(v^{2}+G / x\right) \dot{u}^{2}=\epsilon,
$$



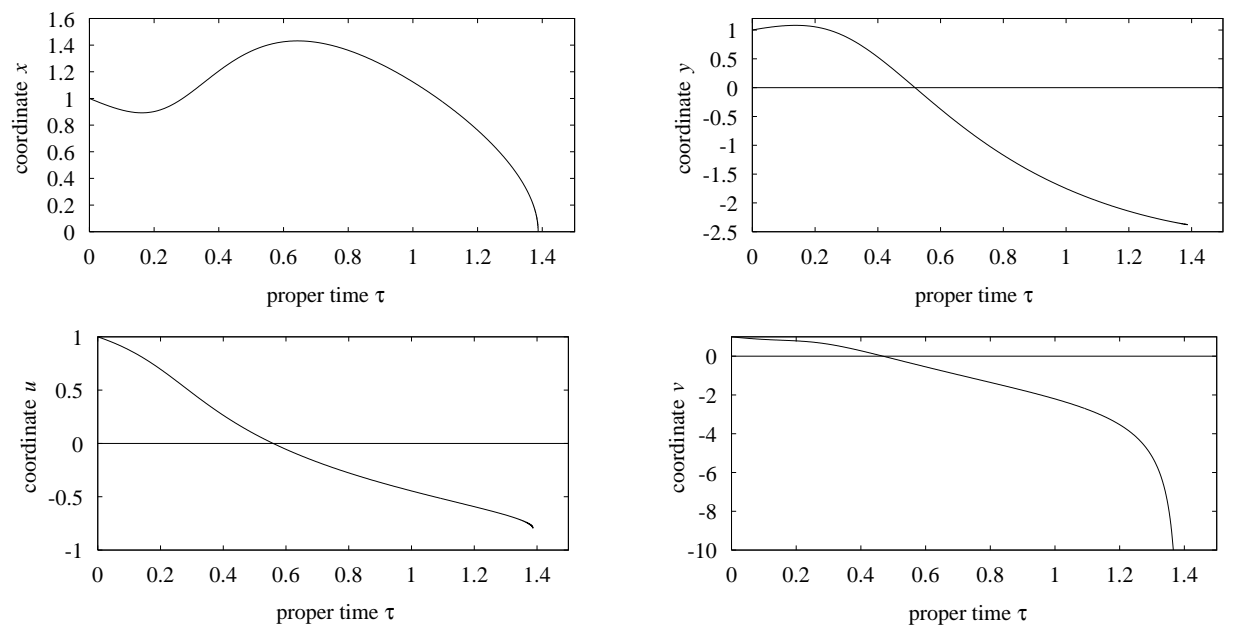

Figure 2: Typical behaviour of timelike geodesics in the Kundt wave spacetime $n=2$. The initial values are $x=y=u=v=1, \dot{x}=\dot{u}=-1, \dot{y}=1, \dot{v}=-2.75$ at $\tau=0$.

where $\epsilon$ denotes the character of the geodesic, namely

$$
\epsilon=\left\{\begin{array} { r } 
{ - 1 } \\
{ 0 } \\
{ + 1 }
\end{array} \text { for } \left\{\begin{array}{l}
\text { timelike } \\
\text { null } \\
\text { spacelike }
\end{array}\right.\right. \text { geodesics. }
$$

Specific geodesic motion given by equations (16)-(19) depends on the particular spacetime function $G(x, y, u)$. We concentrate here on type $N$ vacuum Kundt waves given by the expression (6). If the function $f(\zeta, u)$ is linear in $\zeta$, this generates only a flat space, cf. (44). The simplest radiative spacetimes thus arise when $f=d(u) \zeta^{n}, n=2,3,4, \ldots$. For a real function $d(u)$, in coordinates $x, y$ this corresponds to

$$
G^{(n)}=c(u) \operatorname{Re}\left\{(x+\mathrm{i} y)^{n}\right\} .
$$

An arbitrary function $c(u)$ determines the profile of gravitational wave. Various sandwich and impulsive waves can thus be constructed [34]. However, in our contribution we only consider spacetimes for which the function $G$ is independent of $u$, i.e. $c(u)=$ const. Without loss of generality we assume $c=1$. Indeed, employing the scaling freedom of the metric (8),$u \rightarrow \tilde{u}=\lambda u$, $v \rightarrow \tilde{v}=\lambda^{-1} v, G \rightarrow \tilde{G}=\lambda^{-2} G$, the factor $c$ can be set to unity by an appropriate choice of $\lambda$ ( $G<0$ corresponding to $c=-1$ is related to $G>0$ with $c=1$ by the reflection $x \rightarrow-x$ ).

Even after these simplifications are made, it is difficult to find explicit solutions of equations (16)-(19). To obtain more general insight into the geodesic motion it is necessary to perform numerical simulations. For example, typical behaviour of a test particle in the Kundt spacetime with $G^{(2)}=x^{2}-y^{2}$ is indicated in figure 2 It reaches the envelope singularity $x=0$ in a finite value of the proper time $\tau$, with the remaining coordinates decreasing. However, for different initial values the coordinate $x$ may grow to infinity. Discussion of our numerical studies will be presented elsewhere [35]. Instead, we concentrate here on derivation of families of explicit geodesics which could be used for analytic investigation of the singularities.

\subsection{Explicit geodesics in the hypersurface $y=0$}

The equations simplify if we restrict to geodesics in the hypersurface $y=0$. In fact, it is a natural assumption: in the background Minkowski coordinates this corresponds to $Y=0$ which 
is a plane perpendicular to the symmetry axis of the cylindrical envelope singularity, see figure 1. The expressions (21) for $G^{(n)}$ (with $c=1$ ) and its derivatives on $y=0$ simplify to

$$
G^{(n)}=x^{n}, \quad G_{, x}^{(n)}=n x^{n-1}, \quad G_{, y}^{(n)}=0 .
$$

The equation (17) is then identically satisfied by $y(\tau) \equiv 0$. Remaining equations (16), (19), (18) take the form

$$
\begin{aligned}
\ddot{x}+4 x \dot{v} \dot{u}-\left[4 x v^{2}+2(n+1) x^{n}\right] \dot{u}^{2} & =0, \\
\dot{x}^{2}-4 x^{2} \dot{u} \dot{v}+4 x^{2}\left(v^{2}+x^{n-1}\right) \dot{u}^{2} & =\epsilon, \\
\ddot{u}+2(\dot{x} / x) \dot{u}+2 v \dot{u}^{2} & =0 .
\end{aligned}
$$

From the relation (25) we easily express $v$ (assuming $\dot{u} \neq 0$ ) and substitute into (23) and (24). We obtain a system for the functions $\dot{u}$ and $x$ only,

$$
\begin{aligned}
& 2 \frac{\dddot{u}}{\dot{u}}-3 \frac{\ddot{u}^{2}}{\dot{u}^{2}}+2(n+1) x^{n-1} \dot{u}^{2}+3 \frac{\ddot{x}}{x}=0, \\
& 2 \frac{\dddot{u}}{\dot{u}}-3 \frac{\ddot{u}^{2}}{\dot{u}^{2}}+4 x^{n-1} \dot{u}^{2}+\frac{\dot{x}^{2}}{x^{2}}+4 \frac{\ddot{x}}{x}=\frac{\epsilon}{x^{2}} .
\end{aligned}
$$

Subtracting (27) from (26) we derive an important relation

$$
\left(\frac{1}{2} x^{2}\right)^{\cdot .} \equiv x \ddot{x}+\dot{x}^{2}=\epsilon+2(n-1) x^{n+1} \dot{u}^{2} .
$$

It is possible to express $\dot{u}$ and substitute into (26) but the resulting fourth-order non-linear equation for $x$ is very complicated, and additional assumptions are thus necessary. The term on the right-hand side of (28) indicates that the relation between $\dot{u}$ and powers of $x$ is essential for the character of solution. For example, if $x^{n+1} \dot{u}^{2} \rightarrow 0$ then it becomes $\left(x^{2}\right)^{\cdot *} \approx 2 \epsilon$, which implies

$$
x \approx \sqrt{\epsilon \tau^{2}+C_{1} \tau+C_{0}},
$$

where $C_{0}, C_{1}$ are constants. Such geodesics are possible: figure 3 shows null geodesics $(\epsilon=0)$ obtained by numerical simulations. Linear behaviour of the function $x^{2}$ near $x=0$ is obvious.
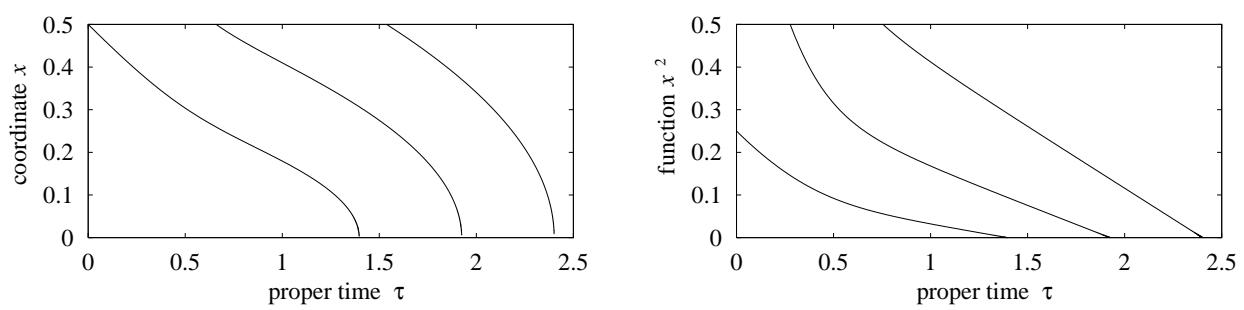

Figure 3: Typical null geodesics approaching the singularity at $x=0$ in the Kundt spacetime $n=2$. The initial values at $\tau=0$ are $x=0.5,1,1.5$, and $\dot{u}=-0.45,-1.2,-2.17$, respectively.

To make the analysis more systematic let us assume a power-law relation between $\dot{u}$ and $x$,

$$
\dot{u}=A x^{a}
$$

where $a, A \neq 0$ are constants. Putting this into (28) we get equation which can be integrated to

$$
\dot{x}^{2}=\epsilon+\frac{4 A^{2}(n-1)}{2 a+n+3} x^{2 a+n+1}+\frac{C}{x^{2}},
$$


with $C$ being the constant of integration. By substituting into (26) we derive the relation

$$
-\epsilon T_{1}+4 A^{2} T_{2} x^{2 a+n+1}+T_{3} \frac{C}{x^{2}}=0
$$

where the constant parameters are

$$
\begin{aligned}
& T_{1}=a(a+2), \\
& T_{2}=\frac{a+n+1}{2 a+n+3}[a(n-1)+2 n], \\
& T_{3}=1-(2+a)^{2} .
\end{aligned}
$$

The equation (32) is exactly satisfied if $\epsilon=0, C=0, T_{2}=0$, or if $\epsilon= \pm 1, C=0,2 a+n+1=0$, i.e. for three possible choices of the parameter $a$,

$$
\begin{array}{ll}
\text { (A) } & a=-(n+1), \\
\text { (B) } & a=-2 n /(n-1), \\
\text { (C) } & a=-(n+1) / 2 .
\end{array}
$$

These cases enable three families of exact geodesics, denoted as $\mathrm{A}^{(n)}, \mathrm{B}^{(n)}$ and $\mathrm{C}^{(n)}$, which will be discussed in detail in section 3.3. Before this, however, we first investigate a general asymptotic behaviour of solutions to (311), (32) as $x \rightarrow 0$ or $x \rightarrow \infty$.

\subsection{Asymptotic behaviour of geodesics}

We start with asymptotic solutions as $x \rightarrow 0$. To avoid the divergence in the term $x^{-2}$ in (32) it is necessary to set either $C=0$ or $T_{3}=0$. All possibilities, summarized in table 1, are:

As1. The region $x \rightarrow 0$ is reached when $C=0, \epsilon=0$, and $2 a+n+1 \equiv k>0$. These conditions imply $x^{n+1} \dot{u}^{2} \rightarrow 0$, i.e. near $x=0$ the solution approaches (29).

As2. For $a=-2$ we obtain $T_{1}=0$. The region $x \rightarrow 0$ can only be approached asymptotically when $C=0, n>3, \epsilon=0$, and the right-hand side of (31) is positive. This is a particular solution corresponding to the choice $k=n-3$ in As1

As3. The choice $a=-1$ implies $T_{3}=0$, and only for $\epsilon=0$ the equation (32) is fulfilled as $x \rightarrow 0$. The right-hand side of equation (B1) is then positive and it can be solved if $C>0$. This asymptotic solution exactly corresponds to (29).

As4. We can also make $T_{3}=0$ by choosing $a=-3$. Remaining conditions are the same as in the case As3 but we have to assume $n>5$ to reach $x \rightarrow 0$. In contrast to As3. $T_{2}=2-n<0$. This solution also corresponds to (29) asymptotically.

\begin{tabular}{|c||c|c|c|c|}
\hline & $C$ & $a$ & Eq. (32) & Eq. (31) \\
\hline \hline As1 & 0 & $\frac{1}{2}(k-n-1)$ & $x^{k} \sim 0, k>0$ & $\dot{x}^{2}=4 A^{2} \frac{n-1}{k+2} x^{k}$ \\
\hline \hline As2 & 0 & -2 & $x^{n-3} \sim 0, n>3$ & $\dot{x}^{2}=4 A^{2} x^{n-3}$ \\
\hline \hline As3 & $>0$ & -1 & $x^{n-1} \sim 0$ & $\dot{x}^{2} \approx C x^{-2}$ \\
\hline \hline As4 & $>0$ & -3 & $x^{n-5} \sim 0, n>5$ & $\dot{x}^{2} \approx C x^{-2}$ \\
\hline \hline
\end{tabular}

Table 1: Asymptotic solutions as $x \rightarrow 0$, assuming $\dot{u}=A x^{a}$. All these geodesics are null $(\epsilon=0)$. 
Explicit forms of $x(\tau)$ corresponding to the above four possibilities are summarized in table2, Notice that the solutions As1 with $0<k<2$ reach $x=0$ in the finite value $\tau_{0}$ of the affine parameter, whereas for $k>2$ they approach this singularity as $\tau \rightarrow \infty$. The solution As2 exhibits an analogous behaviour, according to whether $3<n<5$ or $n>5$.

\begin{tabular}{|c||c|}
\hline & explicit solutions as $x \rightarrow 0$ \\
\hline \hline As1 & $x \approx\left[|2-k| \sqrt{\frac{n-1}{k+2}} A\left(\tau-\tau_{0}\right)\right]^{\frac{2}{2-k}}$ \\
\hline \hline As2 & $x \approx\left[|5-n| A\left(\tau-\tau_{0}\right)\right]^{\frac{2}{5-n}}$ \\
\hline \hline As3 & $x \approx\left[2 \sqrt{C}\left(\tau-\tau_{0}\right)\right]^{\frac{1}{2}}$ \\
\hline As4 & $x \approx\left[2 \sqrt{C}\left(\tau-\tau_{0}\right)\right]^{\frac{1}{2}}$ \\
\hline
\end{tabular}

Table 2: Explicit asymptotic forms of $x(\tau)$ for null geodesics as $x \rightarrow 0$.

We similarly investigate asymptotic geodesics in the region $x \rightarrow \infty$. It is not now in general necessary to set $C=0$ since the term $C x^{-2}$ in (31), (32) becomes negligible for large values of $x$. Specific possibilities, summarised in table 3 are:

As5. The region $x \rightarrow \infty$ is reached asymptotically if $2 a+n+1 \equiv k<0$ and $\epsilon=0$. The case $k=-2$ is, however, forbidden.

As6. The choice $a=-2$ implies $T_{1}=0$, which requires $n=2$ and $\epsilon=0$ to reach the area $x \rightarrow \infty$. This is a particular case of As5 for $k=-1$.

As7. If $a=-3$ then $T_{3}=0$. Asymptotic solutions $x \rightarrow \infty$ now require $n=4$ with $\epsilon=0$. The alternative case $n=2$ corresponds to As5 for $k=-3$.

As8. The choice (A) given by (34) implies $T_{2}=0$. We thus have to put $\epsilon=0$.

As9. Choosing (B), i.e. (35), we also obtain $T_{2}=0$ which implies $\epsilon=0, n \neq 3$.

As10. Finally, the class (C) given by (36) (corresponding to $k=0$ ) is the only one which admits $\epsilon= \pm 1$. The Eq. (32) requires $2 A^{2}=-\epsilon(n-3) /\left(n^{2}-4 n-1\right)$ which excludes $n=3$. The case $n=2$ is inconsistent with Eq. (31), unless $\epsilon=+1, x<0$. For $n=5,6, \ldots$ the geodesics are timelike, for $n=4$ it is a spacelike geodesic.

\begin{tabular}{|c||c|c|c|}
\hline & $a$ & Eq. (32) & Eq. (31) \\
\hline \hline As5 & $\frac{1}{2}(k-n-1)$ & $4 A^{2} T_{2} x^{k}+C T_{3} x^{-2} \approx 0, k<0$ & $\dot{x}^{2}=4 A^{2} \frac{n-1}{k+2} x^{k}+C x^{-2}$ \\
\hline \hline As6 & -2 & $1 / x \sim 0 \quad$ for $n=2$ & $\dot{x}^{2} \approx 4 A^{2} / x$ \\
\hline As7 & -3 & $1 / x \sim 0$ for $n=4$ & $\dot{x}^{2} \approx 12 A^{2} / x$ \\
\hline \hline As8 & $-(n+1)$ & $C x^{-2} \sim 0$ & $\dot{x}^{2}=-4 A^{2} x^{-(n+1)}+C x^{-2}$ \\
\hline As9 & $-2 n /(n-1)$ & $C x^{-2} \sim 0, n \neq 3$ & $\dot{x}^{2}=\frac{4 A^{2}(n-1)^{2}}{(n+1)(n-3)} x^{\frac{n^{2}-4 n-1}{n-1}}+C x^{-2}$ \\
\hline As10 & $-\frac{1}{2}(n+1)$ & $C x^{-2} \sim 0, n \neq 3$ & $\dot{x}^{2}=\frac{-4 \epsilon}{n^{2}-4 n-1}+C x^{-2}$ \\
\hline \hline
\end{tabular}

Table 3: Asymptotic solutions of the form $\dot{u}=A x^{a}$ as $x \rightarrow \infty$ admit $C \neq 0$. All geodesics are null $(\epsilon=0)$, except in the last case As10 for which $\epsilon \neq 0$.

The corresponding asymptotic forms of $x(\tau)$ near $x=\infty$ are summarized in table 4 . 


\begin{tabular}{|c|c|}
\hline & explicit solutions as $x \rightarrow \infty$ \\
\hline As5 & $\begin{array}{lr}x \approx\left[(2-k) \sqrt{\frac{n-1}{k+2}} A\left(\tau-\tau_{0}\right)\right]^{\frac{2}{2-k}} \text { for }-2<k<0 \\
x \approx\left[2 \sqrt{C}\left(\tau-\tau_{0}\right)\right]^{\frac{1}{2}} & \text { for } k<-2\end{array}$ \\
\hline As6 & $x \approx\left[3 A\left(\tau-\tau_{0}\right)\right]^{\frac{2}{3}}$ \\
\hline As7 & $x \approx\left[3 \sqrt{3} A\left(\tau-\tau_{0}\right)\right]^{\frac{2}{3}}$ \\
\hline As8 & 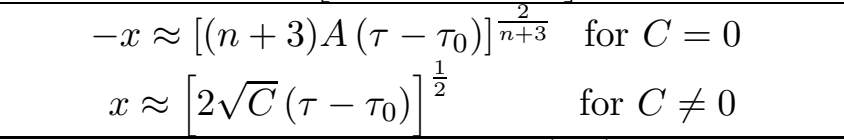 \\
\hline As9 & $\begin{array}{l}x \approx\left[-A \frac{n^{2}-6 n+1}{\sqrt{(n+1)(n-3)}}\left(\tau-\tau_{0}\right)\right]^{\frac{2(1-n)}{n^{2}-6 n+1}} \text { for } n>3 \\
x \approx\left[2 \sqrt{C}\left(\tau-\tau_{0}\right)\right]^{\frac{1}{2}} \quad \text { for } n=2, C \neq 0\end{array}$ \\
\hline As10 & $x \approx \sqrt{\frac{-4 \epsilon}{n^{2}-4 n-1}}\left(\tau-\tau_{0}\right)$ \\
\hline
\end{tabular}

Table 4: Explicit asymptotic geodesics as $x(\tau) \rightarrow \infty$.

Let us note that there exist some special non-trivial exact solutions. For example, the particular case $n=2$ of As8 implies $T_{3}=0$, see (33), and the normalization condition (32) is thus exactly satisfied. The corresponding null geodesics are given by

$$
\sqrt{C x^{2}-4 A^{2} x}\left(\frac{x}{2 C}+\frac{3 A^{2}}{C^{2}}\right)+\frac{6 A^{4}}{C^{5 / 2}} \ln \left(\sqrt{C x^{2}-4 A^{2} x}+x \sqrt{C}-\frac{2 A^{2}}{\sqrt{C}}\right)=\tau-\tau_{0},
$$

where $C>4 A^{2} / x>0$. For large $x$ this approaches the asymptotic form presented in table 4

Similarly, the case $n=5$ of As10 gives timelike geodesics $(\epsilon=-1)$ with $C>0$,

$$
x=\sqrt{\left(\tau-\tau_{0}\right)^{2} \pm 2 \sqrt{C}\left(\tau-\tau_{0}\right)} .
$$

This resembles the asymptotic solution (29) but there is a different sign of the term $\left(\tau-\tau_{0}\right)^{2}$.

It follows from tables 104 that the assumption (30) admits a large class of asymptotic null geodesics on the hypersurface $y=0$, in particular in the region $x \rightarrow \infty$ where the family is parametrized by four constants of integration, namely $k, A, C, \tau_{0}$, and $u_{0}$. The parameter $a$ is in general negative (except for the possibility As1) so that $\dot{u} \rightarrow \infty$ as $x \rightarrow 0$, and $\dot{u} \rightarrow 0$ in the region $x \rightarrow \infty$. Therefore, $u \rightarrow u_{0}$ as $x \rightarrow \infty$ which means that the geodesics become asymptotically tangent to the corresponding wave surface. Numerical simulations also indicate that near $x \rightarrow 0$ the possibility As3 corresponding to $a=-1$ (i.e. $\dot{u} \sim 1 / x$ ) is preferred.

\subsection{Exact "power-law" geodesics}

We have already mentioned that there exist three explicit families $\mathrm{A}^{(n)}, \mathrm{B}^{(n)}, \mathrm{C}^{(n)}$ of exact solutions to the geodesic equations (23)-(25) with $G$ given by (22). These are particular subclasses $C=0$ of As8 As9, As10 respectively, cf. (34)-(36) and table 3. In view of Eq. (31), the coordinate $x$ must be some power of the affine parameter $\tau$. It then follows from Eqs. (30) and (25) that also the coordinates $u$ and $v$ must have the same behaviour, i.e.

$$
x=\alpha\left(\tau-\tau_{0}\right)^{p}, \quad u=\beta\left(\tau-\tau_{0}\right)^{q}+u_{0}, \quad v=\gamma\left(\tau-\tau_{0}\right)^{r} .
$$


The constants $\alpha, \beta, \gamma, p, q, r, \tau_{0}, u_{0}$, related to previous parameters by

$$
a=(q-1) / p, \quad A=q \beta / \alpha^{a},
$$

are restricted by the above equations. A straightforward calculation shows that there are three distinct possibilities, according to the value of the parameter $B \equiv \beta \gamma$ :

$\mathrm{A}^{(n)}$ These solutions which exist for all powers $n$ are given by $B=-1$, but $\alpha^{n-1}<0$ so that this only apply to even $n$ (implying $x<0$ ). The geodesics $A^{(n)}$ are null and correspond to As 8 . characterized by $a=-(n+1)$, with the choice $C=0$.

$\mathrm{B}^{(n)}$ The solutions $\mathrm{B}^{(n)}$ arise when $B=-2 /(n-1)^{2}$ and correspond to the $C=0$ subcase of As9, given by $a=-2 n /(n-1)$. These geodesics are also null in the region $x<0$ for $n=2$, or in the region $x>0$ if $n>3$.

$\mathrm{C}^{(n)}$ The solutions $\mathrm{C}^{(n)}$ for $B=-\frac{1}{2}(n-3) /(n-1)$ in the same domains of $x$ as $\mathrm{B}^{(n)}$ are spacelike $(\epsilon=1)$ for $n=2$ or 4 , and timelike $(\epsilon=-1)$ for $n>4$. This is the possibility As10, characterized by $a=-(n+1) / 2$, for $C=0$. The parameter $\gamma$ is now uniquely given as $2 \gamma^{2}=(n-3) \alpha^{n-1}$. For $n=2$ this implies $\alpha<0$, i.e. $x<0$.

\begin{tabular}{|c||c|c|c|c|c|c|}
\hline & $B$ & $\epsilon$ & $p$ & $q=-r$ & $\alpha^{n-1}$ & $\beta$ \\
\hline \hline $\mathrm{A}^{(n)}$ & -1 & 0 & $\frac{2}{n+3}$ & $-\frac{n-1}{n+3}$ & $-\frac{\gamma^{2}}{(n-1)^{2}}$ & $-\frac{1}{\gamma}$ \\
\hline $\mathrm{B}^{(n)}$ & $\frac{-2}{(n-1)^{2}}$ & 0 & $\frac{2(1-n)}{n^{2}-6 n+1}$ & $\frac{(n-1)^{2}}{n^{2}-6 n+1}$ & $\frac{\gamma^{2}}{4}(n-3)(n+1)$ & $\frac{-2}{\gamma(n-1)^{2}}$ \\
\hline $\mathrm{C}^{(n)}$ & $\frac{-(n-3)}{2(n-1)}$ & \pm 1 & 1 & $-\frac{n-1}{2}$ & $\left(\frac{-4 \epsilon}{n^{2}-4 n-1}\right)^{\frac{n-1}{2}}$ & $\frac{-1}{2 \gamma} \frac{n-3}{n-1}$ \\
\hline
\end{tabular}

Table 5: Three classes of exact solutions in the hypersurface $y=0$. The families $\mathrm{A}^{(n)}, \mathrm{B}^{(n)}$ of null geodesics are parameterised by an arbitrary constant $\gamma$.

Finally, we also attempted to find more general geodesics such that $y \neq 0$. Assuming

$$
x=\alpha\left(\tau-\tau_{0}\right)^{p}, \quad u=\beta\left(\tau-\tau_{0}\right)^{q}+u_{0}, \quad v=\gamma\left(\tau-\tau_{0}\right)^{r}, \quad y=\delta\left(\tau-\tau_{0}\right)^{s},
$$

we found that there are no such geodesics when $n>2$. However, for $n=2$ (i.e. $G^{(2)}=x^{2}-y^{2}$ ) there exists a class of exact null geodesics

$$
x=\frac{3}{2} \gamma^{2}\left(\tau-\tau_{0}\right)^{\frac{2}{5}}, v=\gamma\left(\tau-\tau_{0}\right)^{\frac{1}{5}}, u=-\frac{1}{\gamma}\left(\tau-\tau_{0}\right)^{-\frac{1}{5}}+u_{0}, y= \pm \frac{3 \sqrt{5}}{2} \gamma^{2}\left(\tau-\tau_{0}\right)^{\frac{2}{5}} .
$$

\section{Parallelly transported frames}

After analyzing privileged families of geodesics in the Kundt spacetimes we proceed to the study of singularities at $x=0$ and $x=\infty$. Their character is not obvious: they cannot be scalar curvature singularities $[28,29]$. However, this leaves open the possibility that these are either non-scalar curvature singularities or quasi-regular singularities, according to the classification scheme introduced in [36]. To resolve this problem it is necessary to find orthonormal tetrads which are parallelly transported along geodesics approaching $x=0$ and $x=\infty$. Projections of the curvature tensor onto these frames will then elucidate the character of the singularities. 


\subsection{Frames parallelly transported along any timelike geodesic}

A natural "interpretation" orthonormal frame $\left\{\boldsymbol{e}_{(i)}(\tau)\right\}$ for the Kundt spacetime (8),

$$
\begin{aligned}
e_{(0)}^{\mu} & =u^{\mu}=(\dot{v}, \dot{x}, \dot{y}, \dot{u}), \\
e_{(1)}^{\mu} & =-\left(\dot{x} /\left(2 x^{2} \dot{u}\right), 1,0,0\right), \\
e_{(2)}^{\mu} & =-\left(\dot{y} /\left(2 x^{2} \dot{u}\right), 0,1,0\right), \\
e_{(3)}^{\mu} & =-u^{\mu}+\left(1 /\left(2 x^{2} \dot{u}\right), 0,0,0\right),
\end{aligned}
$$

was introduced and employed in [31]. Mutually perpendicular spatial unit vectors $\boldsymbol{e}_{(1)}, \boldsymbol{e}_{(2)}, \boldsymbol{e}_{(3)}$ are orthogonal to the four-velocity $\mathbf{u}$ of a timelike geodesic observer. Moreover, the vector $\boldsymbol{e}_{(3)}$ is the projection of the principal null direction $\mathbf{k}$. Equation of geodesic deviation expressed in the tetrad (43), see (61) below, exhibits the transverse character of gravitational waves with $\boldsymbol{e}_{(3)}$ representing their direction of propagation (see [31] for more details).

However, in general the tetrad (43) is not parallelly transported. Nevertheless, orthonormal frame $\left\{\boldsymbol{e}_{(i)}^{\|}(\tau)\right\}$ transported parallelly along any geodesic must exist, and at each event it has to be related to the frame (433) by a 3-dimensional rotation of its spatial part. We may naturally assume that such relation is parametrized by the Euler angles $\varphi, \vartheta, \psi$,

$$
\left(\begin{array}{l}
\boldsymbol{e}^{\|}(1) \\
\boldsymbol{e}_{(2)}^{\|} \\
\boldsymbol{e}_{(3)}^{\|}
\end{array}\right)=\left(\begin{array}{ccc}
\cos \varphi & -\sin \varphi & 0 \\
\sin \varphi & \cos \varphi & 0 \\
0 & 0 & 1
\end{array}\right)\left(\begin{array}{ccc}
1 & 0 & 0 \\
0 & \cos \vartheta & -\sin \vartheta \\
0 & \sin \vartheta & \cos \vartheta
\end{array}\right)\left(\begin{array}{ccc}
\cos \psi & -\sin \psi & 0 \\
\sin \psi & \cos \psi & 0 \\
0 & 0 & 1
\end{array}\right)\left(\begin{array}{l}
\boldsymbol{e}_{(1)} \\
\boldsymbol{e}_{(2)} \\
\boldsymbol{e}_{(3)}
\end{array}\right) .
$$

Now, we impose the conditions for the parallel transport of the vectors $\boldsymbol{e}^{\|}(i)(\tau)$. After straight-

forward but somewhat lengthy calculation, using (9) and Eqs. (16)-(18), we obtain the following system of differential equations for the Euler angles,

$$
\begin{aligned}
x \sin \vartheta \dot{\varphi} & =-\cos \psi, \\
x \dot{\vartheta} & =\sin \psi, \\
x \sin \vartheta \dot{\psi} & =-\dot{y} \sin \vartheta+\cos \vartheta \cos \psi .
\end{aligned}
$$

For a given timelike geodesic, characterized by specific functions $x(\tau), y(\tau)$, we thus obtain a specific rotation described by $\varphi(\tau), \vartheta(\tau), \psi(\tau)$ which define the parallelly transported orthonormal frame $\left\{\boldsymbol{e}^{\|}(i)(\tau)\right\}$ rotating with respect to (43).

Interestingly, the situation simplifies considerably for geodesics restricted to $y=0$. The vector $e_{(2)}^{\mu}=(0,0,-1,0)$ is orthogonal to this hypersurface and it is parallelly transported. It only remains to find a suitable rotation of the vectors $\boldsymbol{e}_{(1)}, \boldsymbol{e}_{(3)}$, which may be obtained from (45) for a particular choice $\vartheta=\pi / 2, \psi=\pi$, implying

$$
\dot{\varphi}=x^{-1}(\tau) \text {. }
$$

The parallelly transported orthonormal frame is thus given by

$$
\begin{aligned}
& \boldsymbol{e}_{(1)}^{\|}=-\cos \varphi \boldsymbol{e}_{(1)}+\sin \varphi \boldsymbol{e}_{(3)}, \\
& \boldsymbol{e}_{(2)}^{\|}=-\sin \varphi \boldsymbol{e}_{(1)}-\cos \varphi \boldsymbol{e}_{(3)}, \\
& \boldsymbol{e}_{(3)}^{\|}=-\boldsymbol{e}_{(2)}, \quad \boldsymbol{e}_{(0)}^{\|}=\mathbf{u},
\end{aligned}
$$


or explicitly

$$
\begin{aligned}
& e^{\|_{(1)}^{\mu}}=\cos \varphi\left(\dot{x} /\left(2 x^{2} \dot{u}\right), 1,0,0\right)-\sin \varphi\left(\dot{v}-1 /\left(2 x^{2} \dot{u}\right), \dot{x}, 0, \dot{u}\right), \\
& e^{\|}{ }_{(2)}^{\mu}=\sin \varphi\left(\dot{x} /\left(2 x^{2} \dot{u}\right), 1,0,0\right)+\cos \varphi\left(\dot{v}-1 /\left(2 x^{2} \dot{u}\right), \dot{x}, 0, \dot{u}\right), \\
& e^{\|}{ }_{(3)}^{\mu}=(0,0,1,0), \quad \|_{(0)}^{\mu}=(\dot{v}, \dot{x}, 0, \dot{u}) .
\end{aligned}
$$

The vectors $\boldsymbol{e}_{(1)}, \boldsymbol{e}_{(3)}$ of (43) - the latter indicating the direction of propagation of gravitational waves - rotate with respect to parallelly transported frames along any timelike geodesic, where the angle $\varphi$ is obtained by integration of Eq. (46). Notice that $\dot{\varphi} \rightarrow \infty$ as $x \rightarrow 0$.

\subsection{Frames parallelly transported along any null geodesic}

In order to find parallel frames along null geodesics it is more convenient to employ the formalism of null complex tetrads $\{\mathbf{k}, \mathbf{l}, \mathbf{m}, \overline{\mathbf{m}}\}$ such that $\mathbf{k} \cdot \mathbf{l}=-1, \mathbf{m} \cdot \overline{\mathbf{m}}=1$, see, e.g., [4]. We may start with the natural tetrad (3) and perform its boost followed by a null rotation with fixed $\mathbf{l}$,

$$
\begin{aligned}
\mathbf{k}^{\prime} & =B \mathbf{k}+\bar{K} \mathbf{m}+K \overline{\mathbf{m}}+K \bar{K} B^{-1} \mathbf{l} \\
\mathbf{l}^{\prime} & =B^{-1} \mathbf{l} \\
\mathbf{m}^{\prime} & =\mathbf{m}+K B^{-1} \mathbf{l}
\end{aligned}
$$

where the parameters are given by

$$
K=\dot{\zeta}, \quad B=\dot{v}-F /\left(2 Q^{2}\right) \dot{u}, \quad 2 \dot{\zeta} \dot{\bar{\zeta}}-2 Q^{2} \dot{u} \dot{v}+F \dot{u}^{2}=0
$$

cf. (11). We arrive at the null tetrad

$$
\begin{aligned}
\mathbf{k}^{\prime} & =\dot{v} \partial_{v}+\dot{\zeta} \partial_{\zeta}+\dot{\bar{\zeta}} \partial_{\bar{\zeta}}+\dot{u} \partial_{u}, \\
\mathbf{l}^{\prime} & =F Q^{-2}\left(2 Q^{2} \dot{v}-F \dot{u}\right)^{-1} \partial_{v}+2\left(2 Q^{2} \dot{v}-F \dot{u}\right)^{-1} \partial_{u}, \\
\mathbf{m}^{\prime} & =F Q^{-2} \dot{\zeta}\left(2 Q^{2} \dot{v}-F \dot{u}\right)^{-1} \partial_{v}+\partial_{\bar{\zeta}}+2 \dot{\zeta}\left(2 Q^{2} \dot{v}-F \dot{u}\right)^{-1} \partial_{u},
\end{aligned}
$$

for which the null vector $\mathbf{k}^{\prime}$ is obviously tangent to the corresponding null geodesic and it is, of course, parallelly transported along this. We wish to find a tetrad such that the remaining null vectors are also parallel. This is achieved by performing additional null rotation - this time with fixed $\mathbf{k}^{\prime}$ - and a spatial rotation in the transverse plane,

$$
\begin{aligned}
\mathbf{k} \| & =\mathbf{k}^{\prime}, \\
\mathbf{l}^{\|} & =\mathbf{l}^{\prime}+\bar{L} \mathbf{m}^{\prime}+L \overline{\mathbf{m}}^{\prime}+L \bar{L} \mathbf{k}^{\prime}, \\
\mathbf{m} \| & =\exp (\mathbf{i} \phi)\left(\mathbf{m}^{\prime}+L \mathbf{k}^{\prime}\right) .
\end{aligned}
$$

We have to choose the complex function $L$ and the real function $\phi$ of the affine parameter such that $\mathbf{l}^{\|}, \mathbf{m} \|$ and $\overline{\mathbf{m}} \|$ are parallelly transported. Calculation using (50) and the Christoffel symbols for (11) leads to the system of differential equations

$$
\begin{aligned}
& \dot{\phi}=\mathrm{i} \frac{\dot{\zeta}-\dot{\bar{\zeta}}}{Q}+\frac{\dot{u}^{2}}{2 \dot{\bar{\zeta}}} \mathrm{i}\left[Q\left(\dot{\zeta} H_{, \zeta}-\dot{\bar{\zeta}} H_{, \bar{\zeta}}\right)-(\dot{\zeta}-\dot{\bar{\zeta}}) H\right] \\
& \dot{L}=\left\{\frac{\dot{\zeta}-\dot{\bar{\zeta}}}{Q}+\frac{\dot{u}^{2}}{2 \dot{\dot{\zeta}} \dot{\bar{\zeta}}}\left[Q\left(\dot{\zeta} H_{, \zeta}-\dot{\bar{\zeta}} H_{, \bar{\zeta}}\right)-(\dot{\zeta}-\dot{\bar{\zeta}}) H\right]\right\} L-\frac{1}{Q}-\frac{\dot{u}^{2}}{2 \dot{\bar{\zeta}}}\left(Q H_{, \bar{\zeta}}-H\right) .
\end{aligned}
$$


In real coordinates (17) of the metric (8) these conditions take the form

$$
\begin{aligned}
\dot{\phi} & =-\frac{\dot{y}}{x}-\frac{4 \dot{u}^{2}}{\dot{x}^{2}+\dot{y}^{2}}\left[x\left(\dot{x} G_{, y}-\dot{y} G_{, x}\right)+\dot{y} G\right], \\
\dot{L}_{1}-L_{2} \dot{\phi} & =\frac{2 \sqrt{2} \dot{u}^{2}}{\dot{x}^{2}+\dot{y}^{2}}\left(x G_{, x}-G\right)-\frac{1}{\sqrt{2} x} \\
\dot{L}_{2}+L_{1} \dot{\phi} & =\frac{2 \sqrt{2} \dot{u}^{2}}{\dot{x}^{2}+\dot{y}^{2}} x G_{, y}
\end{aligned}
$$

where $L_{1}, L_{2}$ denote real and imaginary parts of $L, L \equiv L_{1}+\mathrm{i} L_{2}$. These equations are more complicated than the corresponding expressions (45) for parallel frames along timelike geodesics. In particular, they explicitly depend on the structural function $G$.

However, expressions (54) again simplify for null geodesics on the hypersurface $y=0$ in the Kundt spacetimes (22). The simplest parallelly transported frame is then obtained by setting

$$
\phi=0, \quad L_{2}=0, \quad \dot{L}_{1}=2 \sqrt{2}(n-1) x^{n} \dot{x}^{-2} \dot{u}^{2}-(\sqrt{2} x)^{-1} .
$$

In real coordinates $x, y$, the frame is thus explicitly given by

$$
\begin{aligned}
& k^{\|^{\mu}}=(\dot{v}, \dot{x}, 0, \dot{u}), \\
& l^{\mu} \mu\left(L_{1}^{2} \dot{v}+2 \dot{u} \dot{x}^{-2}\left(v^{2}+G / x\right)\left(1+\sqrt{2} L_{1} \dot{x}\right), L_{1}^{2} \dot{x}+\sqrt{2} L_{1},\right. \\
&\left.0, L_{1}^{2} \dot{u}+2 \dot{u} \dot{x}^{-2}\left(1+\sqrt{2} L_{1} \dot{x}\right)\right), \\
& m_{1}^{\|^{\mu}}=\left(L_{1} \dot{v}+\sqrt{2} \dot{u} \dot{x}^{-1}\left(v^{2}+G / x\right), L_{1} \dot{x}+1 / \sqrt{2}, 0, L_{1} \dot{u}+\sqrt{2} \dot{u} \dot{x}^{-1}\right), \\
& m_{2}^{\|^{\mu}}=(0,0,1 / \sqrt{2}, 0) .
\end{aligned}
$$

where $\mathbf{m} \|=\mathbf{m}_{1}^{\|}+\mathbf{i} \mathbf{m}_{2}^{\|}$. For particular null geodesics of the form (30) given by equations (31), (32) with $\epsilon=0, C=0$ we obtain

$$
\dot{L}_{1}=\frac{1}{\sqrt{2}}(n+2 a+2) x^{-1}(\tau) .
$$

This resembles analogous expression (46) for timelike geodesics.

\section{Frame components of curvature tensor and geodesic deviation}

Now we evaluate explicitly the components of the Riemann tensor in the above tetrads which are parallelly transported along timelike or null geodesics in vacuum Kundt spacetimes.

\subsection{Curvature components evaluated along timelike geodesics}

Considering (10), we first project the Riemann tensor onto the "interpretation" frame (43),

$$
\begin{aligned}
R_{(0)(1)(0)(1)}=R_{(0)(1)(1)(3)}=R_{(1)(3)(1)(3)}=\mathcal{A}_{+}, \\
R_{(0)(2)(0)(2)}=R_{(0)(2)(2)(3)}=R_{(2)(3)(2)(3)}=-\mathcal{A}_{+}, \\
R_{(0)(1)(0)(2)}=R_{(0)(1)(2)(3)}=R_{(0)(2)(1)(3)}=R_{(1)(3)(2)(3)}=-\mathcal{A}_{\times},
\end{aligned}
$$

where the amplitudes are

$$
\mathcal{A}_{+}=-2 x \dot{u}^{2} G_{, x x}, \quad \mathcal{A}_{\times}=2 x \dot{u}^{2} G_{, x y},
$$


see also [31]. A straightforward algebraic calculation using the transformation (44) leads to the following non-vanishing components of the curvature tensor in the orthonormal frame $\left\{\boldsymbol{e}_{(i)}(\tau)\right\}$ parallelly transported along a timelike geodesic,

$$
\begin{aligned}
-R_{(2)(3)(2)(3)}^{\|} & =\mathcal{A}_{+}^{\prime}\left(\cos ^{2} \varphi-\sin ^{2} \varphi \cos ^{2} \vartheta\right)+\mathcal{A}_{\times}^{\prime} \cos \vartheta \sin 2 \varphi=R_{(0)(1)(0)(1)}^{\|}, \\
-R_{(0)(1)(2)(3)}^{\|} & =\mathcal{A}_{\times}^{\prime}\left(\cos ^{2} \varphi-\sin ^{2} \varphi \cos ^{2} \vartheta\right)-\mathcal{A}_{+}^{\prime} \cos \vartheta \sin 2 \varphi \\
-R_{(1)(3)(1)(3)}^{\|} & =\mathcal{A}_{+}^{\prime}\left(\sin ^{2} \varphi-\cos ^{2} \varphi \cos ^{2} \vartheta\right)-\mathcal{A}_{\times}^{\prime} \cos \vartheta \sin 2 \varphi=R_{(0)(2)(0)(2)}^{\|}, \\
R_{(0)(2)(1)(3)}^{\|} & =\mathcal{A}_{\times}^{\prime}\left(\sin ^{2} \varphi-\cos ^{2} \varphi \cos ^{2} \vartheta\right)+\mathcal{A}_{+}^{\prime} \cos \vartheta \sin 2 \varphi \\
-R_{(1)(2)(2)(3)}^{\|} & =\mathcal{A}_{+}^{\prime} \frac{1}{2} \sin 2 \vartheta \sin \varphi-\mathcal{A}_{\times}^{\prime} \sin \vartheta \cos \varphi=R_{(0)(1)(0)(3)}^{\|}, \\
-R_{(0)(3)(2)(3)}^{\|} & =\mathcal{A}_{\times}^{\prime} \frac{1}{2} \sin 2 \vartheta \sin \varphi+\mathcal{A}_{+}^{\prime} \sin \vartheta \cos \varphi=-R_{(0)(1)(1)(2)}^{\|}, \\
R_{(0)(3)(1)(3)}^{\|} & =\mathcal{A}_{+}^{\prime} \sin \vartheta \sin \varphi-\mathcal{A}_{\times}^{\prime} \frac{1}{2} \sin 2 \vartheta \cos \varphi=-R_{(0)(2)(1)(2)}^{\|}, \\
-R_{(1)(2)(1)(3)}^{\|} & =\mathcal{A}_{\times}^{\prime} \sin \vartheta \sin \varphi+\mathcal{A}_{+}^{\prime} \frac{1}{2} \sin 2 \vartheta \cos \varphi=-R_{(0)(2)(0)(3)}^{\|}, \\
R_{(1)(3)(2)(3)}^{\|} & =\mathcal{A}_{+}^{\prime} \frac{1}{2}\left(1+\cos ^{2} \vartheta\right) \sin 2 \varphi-\mathcal{A}_{\times}^{\prime} \cos \vartheta \cos 2 \varphi=R_{(0)(1)(0)(2)}^{\|}, \\
-R_{(0)(2)(2)(3)}^{\|} & =\mathcal{A}_{\times}^{\prime} \frac{1}{2}\left(1+\cos ^{2} \vartheta\right) \sin 2 \varphi+\mathcal{A}_{+}^{\prime} \cos \vartheta \cos 2 \varphi=R_{(0)(1)(1)(3)}^{\|}, \\
R_{(1)(2)(1)(2)}^{\|} & =\mathcal{A}_{+}^{\prime} \sin ^{2} \vartheta=-R_{(0)(3)(0)(3)}^{\|}, \\
R_{(0)(3)(1)(2)}^{\|} & =\mathcal{A}_{\times}^{\prime} \sin ^{2} \vartheta
\end{aligned}
$$

where the Euler angles satisfy (45), $\mathcal{A}_{+}^{\prime} \equiv \mathcal{A}_{+} \cos 2 \psi+\mathcal{A}_{\times} \sin 2 \psi, \mathcal{A}_{\times}^{\prime} \equiv-\mathcal{A}_{+} \sin 2 \psi+\mathcal{A}_{\times} \cos 2 \psi$.

The above components of the Riemann tensor can be employed to study geodesic deviation. Let us consider a displacement vector $Z^{\mu}(\tau)$ between two nearby particles moving along timelike geodesics. We project $Z^{\mu}$ onto $\left\{\boldsymbol{e}_{(i)}(\tau)\right\}, Z^{(i)} \equiv e_{\mu}^{(i)} Z^{\mu}$, and denote its absolute derivatives as $\dot{Z}^{(i)} \equiv e_{\mu}^{(i)}\left(\mathrm{D} Z^{\mu} / \mathrm{d} \tau\right), \ddot{Z}^{(i)} \equiv e_{\mu}^{(i)}\left(\mathrm{D}^{2} Z^{\mu} / \mathrm{d}^{2} \tau\right)$. The equation of geodesic deviation with respect to (43) is $\ddot{Z}^{(i)}=-R_{(0)(j)(0)}^{(i)} Z^{(j)}$, i.e. using (58),

$$
\begin{aligned}
& \ddot{Z}^{(1)}=-\mathcal{A}_{+} Z^{(1)}+\mathcal{A}_{\times} Z^{(2)}, \\
& \ddot{Z}^{(2)}=\mathcal{A}_{+} Z^{(2)}+\mathcal{A}_{\times} Z^{(1)}, \\
& \ddot{Z}^{(3)}=0,
\end{aligned}
$$

see [31]. The structure of the right-hand side exhibits the transverse character of gravitational waves with two polarization modes (represented by the amplitudes $\mathcal{A}_{+}, \mathcal{A}_{\times}$) which propagate in the spatial direction of $\mathbf{e}_{(3)}$. However, the left-hand side is complicated because the frame (43) is not parallelly propagated. Nevertheless, using the relation (44), (45) we can express $\ddot{Z}^{(i)}$ in terms of the derivatives $Z^{(i)}$ with respect to $\tau$. The equation (61) thus takes the form

$$
\begin{aligned}
\frac{\mathrm{d}^{2} Z^{(1)}}{\mathrm{d} \tau^{2}}+\frac{2 \dot{y}}{x} \frac{\mathrm{d} Z^{(2)}}{\mathrm{d} \tau}-\frac{2}{x} \frac{\mathrm{d} Z^{(3)}}{\mathrm{d} \tau}= & -\left(\mathcal{A}_{+}-\frac{1}{x^{2}}-\frac{\dot{y}^{2}}{x^{2}}\right) Z^{(1)} \\
& +\left(\mathcal{A}_{\times}+\frac{\dot{y} \dot{x}}{x^{2}}-\frac{\ddot{y}}{x}\right) Z^{(2)}-\frac{\dot{x}}{x^{2}} Z^{(3)}, \\
\frac{\mathrm{d}^{2} Z^{(2)}}{\mathrm{d} \tau^{2}}-\frac{2 \dot{y}}{x} \frac{\mathrm{d} Z^{(1)}}{\mathrm{d} \tau}= & \left(\mathcal{A}_{\times}-\frac{\dot{y} \dot{x}}{x^{2}}+\frac{\ddot{y}}{x}\right) Z^{(1)}, \\
& +\left(\mathcal{A}_{+}+\frac{\dot{y}^{2}}{x^{2}}\right) Z^{(2)}-\frac{\dot{y}}{x^{2}} Z^{(3)},
\end{aligned}
$$




$$
\frac{\mathrm{d}^{2} Z^{(3)}}{\mathrm{d} \tau^{2}}+\frac{2}{x} \frac{\mathrm{d} Z^{(1)}}{\mathrm{d} \tau}=\frac{\dot{x}}{x^{2}} Z^{(1)}-\frac{\dot{y}}{x^{2}} Z^{(2)}+\frac{1}{x^{2}} Z^{(3)}
$$

In the Kundt spacetimes given by $G^{(n)}$ there are timelike geodesics restricted to the privileged hypersurface $y=0$, see (22). In such a case we obtain $\mathcal{A}_{\times}=0, \vartheta=\pi / 2, \psi=\pi$, and $\varphi$ solving (46). The only components (60) of the Riemann tensor with respect to the frame (47) are thus

$$
\begin{aligned}
-R_{(2)(3)(2)(3)}^{\|} & =\mathcal{A}_{+} \cos ^{2} \varphi=R_{(0)(1)(0)(1)}^{\|}, \\
-R_{(1)(3)(1)(3)}^{\|} & =\mathcal{A}_{+} \sin ^{2} \varphi=R_{(0)(2)(0)(2)}^{\|}, \\
-R_{(0)(3)(2)(3)}^{\|} & =\mathcal{A}_{+} \cos \varphi=-R_{(0)(1)(1)(2)}^{\|}, \\
R_{(0)(3)(1)(3)}^{\|} & =\mathcal{A}_{+} \sin \varphi=-R_{(0)(2)(1)(2)}^{\|}, \\
R_{(1)(3)(2)(3)}^{\|} & =\mathcal{A}_{+} \sin \varphi \cos \varphi=R_{(0)(1)(0)(2)}^{\|}, \\
R_{(1)(2)(1)(2)}^{\|} & =\mathcal{A}_{+}=-R_{(0)(3)(0)(3)}^{\|} .
\end{aligned}
$$

The equations of geodesic deviation (62) also considerably simplify since $\dot{y}=0=\mathcal{A}_{\times}$.

\subsection{Curvature components evaluated along null geodesics}

We also calculate components of the Riemann tensor in the parallelly transported null tetrad (52). For vacuum spacetimes the curvature tensor coincides with the Weyl tensor. It is convenient to use standard transformations of the NP coefficients $\Psi_{j}, j=0,1,2,3,4$, see e.g. [4]. We start with the expression (4) with respect to the tetrad (3), and perform boost $B$, null rotation $K$, followed by null rotation $L$ and spatial rotation $\phi$, where these parameters are given by (50) and (53), respectively. We obtain the components,

$$
\Psi_{j}^{\|}=e^{(2-j) \mathrm{i} \phi} K^{4-j}(1+K \bar{L})^{j} \Psi
$$

where, using (150)

$$
\begin{aligned}
\Psi & \equiv B^{-2} \Psi_{4}=\frac{Q \dot{u}^{2}}{2(\dot{\bar{\zeta}})^{2}} H_{, \zeta \zeta} \\
& =\frac{8 x \dot{u}^{2}}{\left(\dot{x}^{2}+\dot{y}^{2}\right)^{2}}\left(-G_{, x x}+i G_{, x y}\right)=4 \frac{\mathcal{A}_{+}+i \mathcal{A}_{\times}}{\left(\dot{x}^{2}+\dot{y}^{2}\right)^{2}} .
\end{aligned}
$$

Comparing (64) with (60) for timelike geodesics (for which only $\mathcal{A}_{+}$and $\mathcal{A}_{\times}$are sufficient to describe the behaviour of gravitational filed), investigation of the curvature tensor along null geodesics is more complicated. One has to study not only $\Psi$ but also various powers of $K$ and

$(1+K \bar{L})$. However, there is a simplification for null geodesic $y=0$ in vacuum Kundt spacetimes with $G^{(n)}$; we obtain $\Psi=4 \mathcal{A}_{+} / \dot{x}^{4}, \phi=0, K=\dot{x} / \sqrt{2}$, and $L=L_{1}$ given by (55), so that

$$
\Psi_{j}^{\|}=\left(L_{1}+\sqrt{2} / \dot{x}\right)^{j} \mathcal{A}_{+}, \quad j=0,1,2,3,4 .
$$

\section{Character of the singularities in the Kundt spacetimes}

Using the above results we will now discuss the behaviour of the curvature tensor components with respect to frames parallelly propagated along timelike or null geodesics. This will elucidate the character on the singularities, in particular of the envelope singularity $x=0$. 
It has been shown that such components of the Riemann tensor are proportional to the amplitudes $\mathcal{A}_{+}, \mathcal{A}_{\times}$, see expressions (60) or (64) for timelike or null geodesics, respectively. We calculate these amplitudes (59) for spacetimes given by the structural function (21) with $c=1$ on the hypersurface $y=0$ as $\mathcal{A}_{+}=-2 n(n-1) x^{n-1} \dot{u}^{2}, \mathcal{A}_{\times}=0$. The behaviour of $\mathcal{A}_{+}$as $x \rightarrow 0$ or $x \rightarrow \infty$ depends on the mutual relation between $\dot{u}$ and the powers of $x$. This again justifies our assumption (30) which enabled us in section 3 to derive particular classes of exact or asymptotic geodesics, summarized in tables 1,5. For all these we obtain

$$
\mathcal{A}_{+}=-2 n(n-1) A^{2} x^{2 a+n-1} .
$$

It is now easy to express explicitly the behaviour of this function for exact geodesics denoted above as $\mathrm{A}^{(n)}, \mathrm{B}^{(n)}, \mathrm{C}^{(n)}$. Recall that only the geodesics $\mathrm{C}^{(n)}$ are timelike if $n>4$, and the behaviour of $\mathcal{A}_{+}$is thus sufficient. All other geodesics are null and for the discussion of (66) it is necessary to evaluate also the function $L_{1}+\sqrt{2} / \dot{x}$. In table [6] we present a summary of the powers of $\left(\tau-\tau_{0}\right)$ corresponding to each quantity.

\begin{tabular}{|c|c||c|c|c|c|}
\hline Class & $\epsilon$ & $x$ & $\mathcal{A}_{+}$ & $L_{1}+\sqrt{2} / \dot{x}$ & $\Psi_{j}^{\|}=\left(L_{1}+\sqrt{2} / \dot{x}\right)^{j} \mathcal{A}_{+}$ \\
\hline \hline $\mathrm{A}^{(n)}$ & 0 & $\frac{2}{n+3}$ & -2 & $\frac{n+1}{n+3}$ & $\frac{(j-2) n+(j-6)}{n+3}$ \\
\hline $\mathrm{B}^{(n)}$ & 0 & $\frac{2(1-n)}{n^{2}-6 n+1}$ & -2 & $\frac{n^{2}-4 n-1}{n^{2}-6 n+1}$ & $\frac{(j-2) n^{2}-4(j-3) n-(j+2)}{n^{2}-6 n+1}$ \\
\hline $\mathrm{C}^{(n)}$ & $\begin{array}{c}-1 \text { if } \\
n>4\end{array}$ & 1 & -2 & - & - \\
\hline
\end{tabular}

Table 6: The powers of $\left(\tau-\tau_{0}\right)$ for various quantities which enter the components of the curvature tensor in frames parallelly propagated along classes of exact geodesics $y=0$.

Interestingly, although the power-law dependence of $x \sim\left(\tau-\tau_{0}\right)^{p}$ on the affine parameter $\tau$ varies for different classes $\mathrm{A}^{(n)}, \mathrm{B}^{(n)}$, and $\mathrm{C}^{(n)}$ of geodesics, the dependence of the curvature tensor amplitude $\mathcal{A}_{+}$is the same, namely

$$
\mathcal{A}_{+} \sim\left(\tau-\tau_{0}\right)^{-2}
$$

This is the consequence of the fact that $2 a+n-1=2(q-1) / p+(n-1)$, cf. (40), and also $q=-(n-1) p / 2$, which can be seen from table [5] so that $2 a+n-1=-2 / p$. Similarly, the relation (57) gives $\dot{L}_{1} \sim x^{-1} \sim\left(\tau-\tau_{0}\right)^{-p}$, implying $L_{1} \sim\left(\tau-\tau_{0}\right)^{1-p}$. Since $\dot{x} \sim\left(\tau-\tau_{0}\right)^{p-1}$, both $L_{1}$ and $1 / \dot{x}$ exhibit the same behaviour,

$$
L_{1}+\sqrt{2} / \dot{x} \sim\left(\tau-\tau_{0}\right)^{1-p}
$$

Let us now discuss specific behaviour of the curvature tensor in some more detail.

The only class which admits timelike geodesics is $\mathrm{C}^{(n)}$ when $n>4$. This is characterized by $a=-(n+1) / 2$ and $p=1$, i.e. $x \sim\left(\tau-\tau_{0}\right)$. The amplitude (68) thus behaves as

$$
\begin{aligned}
& \lim _{x \rightarrow 0} \mathcal{A}_{+} \sim \lim _{\tau \rightarrow \tau_{0}}\left(\tau-\tau_{0}\right)^{-2}=\infty, \\
& \lim _{x \rightarrow \infty} \mathcal{A}_{+} \sim \lim _{\tau \rightarrow \infty}\left(\tau-\tau_{0}\right)^{-2}=0 .
\end{aligned}
$$


Obviously, all non-trivial components (63) of the curvature tensor with respect to frames parallelly transported along these timelike geodesics approaching $x=0$ diverge. The envelope singularity of the radiative Kundt spacetimes is thus a non-scalar curvature singularity which is reached in a finite value of the proper time. On the other hand, the spacetimes become flat in the region $x \rightarrow \infty$ for these timelike geodesics.

Analogous discussion concerning null geodesics is more complicated since the components (66) depend on $\mathcal{A}_{+}$and also on powers of the function $\left(L_{1}+\sqrt{2} / \dot{x}\right)$. The class of exact null geodesics $\mathrm{A}^{(n)}$ is characterized by $a=-(n+1)$ and $p=2 /(n+3)$, so that $x \sim\left(\tau-\tau_{0}\right)^{2 /(n+3)}$, $\mathcal{A}_{+} \sim x^{-(n+3)} \sim\left(\tau-\tau_{0}\right)^{-2}$. The relation (69) reads $\left(L_{1}+\sqrt{2} / \dot{x}\right) \sim\left(\tau-\tau_{0}\right)^{(n+1) /(n+3)}$. The frame components of the curvature tensor are thus

$$
\Psi_{j}^{\|} \sim\left(\tau-\tau_{0}\right)^{[(j-2) n+(j-6)] /(n+3)} .
$$

The lowest power appears for $j=0$, namely $\Psi_{0}^{\|}=\mathcal{A}_{+} \sim\left(\tau-\tau_{0}\right)^{-2}$, whereas the highest one for $j=4, \Psi_{4}^{\|} \sim\left(\tau-\tau_{0}\right)^{2(n-1) /(n+3)}$. Obviously, there is always a curvature singularity at $x=0$ since the frame component of the curvature tensor $\Psi_{0}^{\|}$diverges,

$$
\lim _{x \rightarrow 0} \Psi_{0}^{\|} \sim \lim _{\tau \rightarrow \tau_{0}}\left(\tau-\tau_{0}\right)^{-2}=\infty
$$

Other components also diverge at $x=0$ if the corresponding power in (71) is negative, i.e. for

$$
j<2+\frac{4}{n+1}
$$

Thus, $\Psi_{0}^{\|}, \Psi_{1}^{\|}, \Psi_{2}^{\|}$always diverge, $\Psi_{3}^{\|}$diverges at $x=0$ for spacetime with $n=2$, whereas $\Psi_{4}^{\|}$ always approaches zero there. For null geodesics of this class $\mathrm{A}^{(n)}$ approaching the region $x \rightarrow \infty$ the situation is completely the opposite: $\Psi_{0}^{\|}, \Psi_{1}^{\|}, \Psi_{2}^{\|}$always vanish asymptotically, whereas

$$
\lim _{x \rightarrow \infty} \Psi_{4}^{\|} \sim \lim _{\tau \rightarrow \infty}\left(\tau-\tau_{0}\right)^{2(n-1) /(n+3)}=\infty
$$

Behaviour of the curvature tensor along null geodesics $\mathrm{B}^{(n)}$ with $a=-2 n /(n-1), p=$ $2(1-n) /\left(n^{2}-6 n+1\right)$, depends on the parameter $n$ of the specific function (21) of the spacetime. In particular, $x \sim\left(\tau-\tau_{0}\right)^{p}$ so that

$$
\begin{aligned}
& \text { for } \quad n<6: \quad x \rightarrow 0 \Leftrightarrow \tau \rightarrow \tau_{0}, \quad x \rightarrow \infty \Leftrightarrow \tau \rightarrow \infty \text {, } \\
& \text { for } \quad n \geq 6: \quad x \rightarrow 0 \Leftrightarrow \tau \rightarrow \infty, \quad x \rightarrow \infty \Leftrightarrow \tau \rightarrow \tau_{0} \text {. }
\end{aligned}
$$

The behaviour is thus more complicated. The amplitude $\mathcal{A}_{+}=\Psi_{0}^{\|}$is proportional to $\left(\tau-\tau_{0}\right)^{-2}$, i.e. singular as $\tau \rightarrow \tau_{0}$, other components of the curvature tensor are

$$
\Psi_{j}^{\|} \sim\left(\tau-\tau_{0}\right)^{\left[(j-2) n^{2}-4(j-3) n-(j+2)\right] /\left(n^{2}-6 n+1\right)} .
$$

The frame component diverges as $\tau \rightarrow \tau_{0}$ if the corresponding power of (76), which is equal to $j(1-p)-2$, is negative, i.e. (considering $p<1$ ) when

$$
j<\frac{2}{1-p}=2-\frac{4(n-1)}{n^{2}-4 n-1} .
$$

The behaviour for $\tau \rightarrow \infty$ is exactly the complementary one. In particular, for spacetimes with $n=2$ the components $\Psi_{0}^{\|}, \Psi_{1}^{\|}, \Psi_{2}^{\|}$diverge at $x=0$ whereas $\Psi_{3}^{\|}, \Psi_{4}^{\|}$approach zero there. For 
$n=3$ the components $\Psi_{0}^{\|}, \Psi_{1}^{\|}, \Psi_{2}^{\|}, \Psi_{3}^{\|}$diverge at $x=0$ while $\Psi_{4}^{\|}$is regular, for $n=4$ all the components diverge. In spacetimes $n=5$ with $p=2$ the condition reads $j>-2$ so that all components $\Psi_{j}^{\|}$diverge as $x \rightarrow 0$, but all approach zero as $x \rightarrow \infty$. For $n=6$ the condition (77) is satisfied only for $j=0$ so that, considering (75), $\Psi_{1}^{\|}, \Psi_{2}^{\|}, \Psi_{3}^{\|}, \Psi_{4}^{\|}$diverge at $x=0$, and $\Psi_{0}^{\|}$ diverges as $x \rightarrow \infty$. For spacetimes with very large parameter $n$ the condition is satisfied when $j=0$ or $j=1$ so that $\Psi_{2}^{\|}, \Psi_{3}^{\|}, \Psi_{4}^{\|}$diverge at $x=0$, and $\Psi_{0}^{\|}, \Psi_{1}^{\|}$diverge as $x \rightarrow \infty$.

The above described behaviour of the curvature tensor near $x=0$ and $x=\infty$ has been obtained using explicit privileged classes $\mathrm{A}^{(n)}, \mathrm{B}^{(n)}$, and $\mathrm{C}^{(n)}$ of exact geodesics on the hypersurface $y=0$. This can be confirmed by (the only) "power-law" geodesics of the type (41) which admit $y \neq 0$. These null geodesics in spacetimes with $n=2$ are explicitly given by (42). There is $x \sim\left(\tau-\tau_{0}\right)^{2 / 5} \sim y, u \sim\left(\tau-\tau_{0}\right)^{-1 / 5}$ so that, according to (159), $\mathcal{A}_{\times}=0, \mathcal{A}_{+} \sim x \dot{u}^{2} \sim\left(\tau-\tau_{0}\right)^{-2}$, and $K \sim\left(\tau-\tau_{0}\right)^{-3 / 5},(1+K \bar{L})=$ const. Consequently, from (64) we obtain

$$
\Psi_{j}^{\|} \sim\left(\tau-\tau_{0}\right)^{3 j / 5-2}
$$

which implies a divergence of the components $\Psi_{0}^{\|}, \Psi_{1}^{\|}, \Psi_{2}^{\|}, \Psi_{3}^{\|}$, at $x=0$. On the other hand, $\Psi_{4}^{\|} \sim\left(\tau-\tau_{0}\right)^{2 / 5} \sim x$, which indicates a singularity at $x \rightarrow \infty$.

Finally, we investigate the behaviour of the curvature tensor along more general classes of asymptotic geodesics approaching the regions $x=0$ or $x=\infty$. These have been described in previous section 3.2 The explicit class of null geodesics As1 is characterized by $x \sim\left(\tau-\tau_{0}\right)^{2 /(2-k)}$ where $k=2 a+n+1$, see tables 1 and 2. In view of (67) we obtain

$$
\mathcal{A}_{+} \sim x^{k-2} \sim\left(\tau-\tau_{0}\right)^{-2}
$$

so that the component $\Psi_{0}^{\|}=\mathcal{A}_{+}$diverges at $x=0$ as $\tau \rightarrow \tau_{0}$. Other components are given by $\Psi_{j}^{\|} \sim\left(\tau-\tau_{0}\right)^{[(j-2) k+4] /(k-2)}$. The same applies to asymptotic geodesics As2 which is a particular subcase of As1 for $k=n-3$. The classes As3 and As4 exhibit somewhat different dependence. It turns out that $\Psi_{j}^{\|} \sim\left(\tau-\tau_{0}\right)^{(n-3+j) / 2}$ and $\Psi_{j}^{\|} \sim\left(\tau-\tau_{0}\right)^{(n-7+j) / 2}$, respectively. Therefore, as $x \rightarrow 0$ with $\tau \rightarrow \tau_{0}$, only the component $\Psi_{0}^{\|}$diverges for $n=2$ in case As3 and for $n=6$ in case As4. Other components of the curvature tensor are regular at $x=0$.

Concerning the complementary asymptotics $x \rightarrow \infty$, for the case As5 the functional dependence of $x$ is the same as in the case As1 (cf. table 4) so that the behaviour of the amplitude is also given by (79). With $\tau \rightarrow \infty$ the component $\Psi_{0}^{\|}$vanishes, but $\Psi_{j}^{\|}$diverge when $j>2-4 / k>2$. Since As6 and As7 are particular cases of As5 for $k=-1$, all the components $\Psi_{j}^{\|}$vanish asympotically along these special geodesics. Finally, there are three subclasses As8, As9, As10, For $C=0, x \rightarrow \infty$, they asymptotically approach the exact geodesics $\mathrm{A}^{(n)}, \mathrm{B}^{(n)}, \mathrm{C}^{(n)}$ which we have discussed in detail at the beginning of this section. For $C \neq 0$ there are particular null geodesics of the form $x \sim \sqrt{\tau-\tau_{0}}$, for which $\Psi_{j}^{\|} \sim\left(\tau-\tau_{0}\right)^{(j-n-3) / 2}$ in case As8, and $\Psi_{j}^{\|} \sim\left(\tau-\tau_{0}\right)^{(j-7) / 2}$ in case As9. In both these special cases, all components vanish as $x \rightarrow \infty$, i.e. as $\tau \rightarrow \infty$.

To summarise our somewhat lengthy discussion: typical components (like $\mathcal{A}_{+}=\Psi_{0}^{\|}$) of the Riemann tensor with respect to parallelly propagated frames diverge as $x \rightarrow 0$ (which is reached for $\tau \rightarrow \tau_{0}$ ) both for timelike and null geodesics, see relations (70), (71)-(73), (76), (78), and (179). Concerning the behaviour in the complementary region $x \rightarrow \infty$ of the Kundt spacetimes there are also divergences in other components of the curvature tensor (typically $\Psi_{4}^{\|}$) as $\tau \rightarrow \infty$. However, this is not so along all null geodesics and, in particular, along timelike geodesics of the class $\mathrm{C}^{(n)}$ for which $\mathcal{A}_{+} \rightarrow 0$, see (70). Further analysis is thus required to fully understand the global structure of null and timelike infinities of these spacetimes. 


\section{Conclusions}

In the present work we have studied vacuum solutions to Einstein's equations which represent exact radiative spacetimes of the Kundt type. Our main objective was to investigate the geodesic motion and to elucidate the character of the singularities contained.

We found, in section 3 , particular families of exact geodesics denoted as $\mathrm{A}^{(n)}, \mathrm{B}^{(n)}, \mathrm{C}^{(n)}$ (cf. table [5), and also classes of approximate solutions to the geodesic equations on the hypersurface $y=0$ as $x \rightarrow 0$ (As1 to As4) or $x \rightarrow \infty$ (As5 to As10), see tables 2 and 4 These asymptotic families are quite large as they contain several independent parameters, e.g., $k, A, C, \tau_{0}, u_{0}$.

In section 4 we presented tetrads that are parallelly transported along any timelike or null geodesic. Somewhat surprisingly, these could be given in a closed explicit form. In particular, we demonstrated that the spatial direction $\boldsymbol{e}_{(3)}$ of propagation of the Kundt gravitational wave always rotates with respect to parallelly propagated frames. This is similar to analogous behaviour which was discovered previously in the Siklos spacetimes with $\Lambda<0$ [37]. It can be explained by the fact that both Kundt and Siklos waves belong to a larger class of generalized Kundt spacetimes for which the spin coefficient $\tau$ is non-vanishing [19], see also [5].

Subsequently, in section [5 we calculated projections of the curvature tensor onto the frames that are parallelly propagated along arbitrary timelike or null geodesic. Not only could these components be used for studies of geodesic deviation, see equation (62), but they are also crucial for discussion of the character of singularities.

This is done in the final section [6. From the frame components of the curvature tensor we explicitly demonstrated that there is a physical singularity at $x=0$ which is reached in a finite value of the affine parameter $\tau_{0}$ both for timelike and null geodesics. Although all scalar curvature invariants of these Kundt spacetimes identically vanish, the expanding envelope of the rotated wave surfaces $u=$ const. (see figure 1) that is localized on $x=0$ is a (nonscalar) curvature singularity, according to the classification scheme introduced in [36]. It is not a "mild" quasi-regular singularity because some of the Riemann tensor components in the parallelly propagated orthonormal frames diverge as $x \rightarrow 0$. It is thus not possible to extend the Kundt solution "smoothly" into the inner part of the expanding wave-front envelope $x=0$ by a simple flat Minkowski region.

The complementary region $x \rightarrow \infty$ of the Kundt spacetimes corresponds to conformal infinity because it is reached by geodesics only as $\tau \rightarrow \infty$. There are also divergences in some components of the curvature tensor, even if not along all geodesics. A more detailed analysis is still necessary to understand the global structure of these null and timelike infinities of the Kundt wave spacetimes.

Finally, let us observe that many of the above results obtained for type $N$ vacuum Kundt waves remain valid also for conformally flat spacetimes (5) containing pure radiation. In particular, on the hypersurface $y=0$ the metric function $G_{0}=x^{2}+y^{2}$ (including its first derivatives) is effectively identical to the function (21) with $n=2$, i.e. $G^{(2)}=x^{2}-y^{2}$. We thus obtain the same forms of the geodesics and parallelly propagated frames. The tetrad components of the curvature tensor (63) are also similar, but expressions (64) now vanish identically because the spacetimes are conformally flat $(\Psi=0)$. However, projecting directly (10) onto (56) we obtain, for example, $R_{\alpha \beta \gamma \delta} m_{2}^{\| \alpha} k^{\|^{\beta}} m_{2}^{\| \gamma} k^{\|^{\delta}}=-2 x \dot{u}^{2}=-2 A^{2} x^{2 a+1}$. For the class $\mathrm{A}^{(2)}$ of exact null geodesics one has $a=-3, x \sim\left(\tau-\tau_{0}\right)^{2 / 5}$, for $\mathrm{B}^{(2)}$ there is $a=-4, x \sim\left(\tau-\tau_{0}\right)^{2 / 7}$. In both cases the above frame component of the Riemann tensor is proportional to $\left(\tau-\tau_{0}\right)^{-2}$ and thus diverges as $x \rightarrow 0$ with $\tau \rightarrow \tau_{0}$. Obviously, the envelope singularity $x=0$ in conformally flat Kundt spacetimes with pure radiation is again a non-scalar curvature singularity. 


\section{Acknowledgments}

This work was supported by the grant GACR-202/02/0735 from the Czech Republic and the grant GAUK from the Charles University in Prague. The authors are grateful to M. Ortaggio and J. B. Griffiths for useful comments.

\section{References}

[1] Kundt W 1961 Z. Phys. 16377

[2] Kundt W 1962 Proc. R. Soc. A 270328

[3] Ehlers J and Kundt W 1962 Gravitation: An Introduction to Current Research, ed L Witten (New York: Wiley) p 49

[4] Stephani H, Kramer D, MacCallum M, Hoenselaers C and Herlt E 2003 Exact Solutions to Einstein's Field Equations (Cambridge: Cambridge University Press)

[5] Podolský J and Ortaggio M 2003 Class. Quantum Grav. 201685

[6] Brinkmann H W 1925 Math. Ann. 94119

[7] Baldwin O R and Jeffery G B 1926 Proc. R. Soc. A 11195

[8] Brdička M 1951 Proc. Roy. Irish Acad. A54 137

[9] Bondi H, Pirani F A E and Robinson I 1959 Proc. R. Soc. A 251519

[10] Nariai H 1951 Sci. Rep. Tôhoku Univ. 3562

[11] Levi-Civita T 1917 Rend. Acc. Lincei 26519

[12] Bertotti B 1959 Phys. Rev. 1161331

[13] Robinson I 1959 Bull. Acad. Polon. 7351

[14] Ozsváth I, Robinson I and Rózga K 1985 J. Math. Phys. 261755

[15] García Díaz A and Plebański J F 1981 J. Math. Phys. 222655

[16] Siklos S 1985 Galaxies, Axisymmetric Systems and Relativity, ed M A H MacCallum (Cambridge: Cambridge University Press) pp 247-274

[17] Van den Bergh N, Gunzig E and Nardone P 1990 Class. Quantum Grav. 7 L175

[18] Bičák J and Podolský J 1999 J. Math. Phys. 404495

[19] Griffiths J B, Docherty P and Podolský J 2004 Class. Quantum Grav. 21207

[20] Wils P 1989 Class. Quantum Grav. 61243

[21] Koutras A and McIntosh C 1996 Class. Quantum Grav. 13 L47

[22] Edgar S B and Ludwig G 1997 Class. Quantum Grav. 14 L65

[23] Edgar S B and Ludwig G 1997 Class. Quantum Grav. 143453 
[24] Edgar S B and Ludwig G 1997 Gen. Rel. Grav. 291309

[25] Skea J E F 1997 Class. Quantum Grav. 142393

[26] Griffiths J B and Podolský J 1998 Class. Quantum Grav. 153863

[27] Barnes A 2001 Class. Quantum Grav. 185287

[28] Bičák J and Pravda V 1998 Class. Quantum Grav. 151539

[29] Pravda V, Pravdová A, Coley A and Milson R 2002 Class. Quantum Grav. 196213

[30] Coley A A 2002 Phys. Rev. Lett. 89281601

[31] Bičák J and Podolský J 1999 J. Math. Phys. 404506

[32] Podolský J and Veselý K 1998 Phys. Rev. D 58081501

[33] Urbantke H 1979 J. Math. Phys. 201851

[34] Podolský J 1998 Class. Quantum Grav. 153229

[35] Beláň M and Podolský J in preparation

[36] Ellis G F R and Schmidt B G 1977 Gen. Relativ. Gravit. 8915

[37] Podolský J 1998 Class. Quantum Grav. 15719 